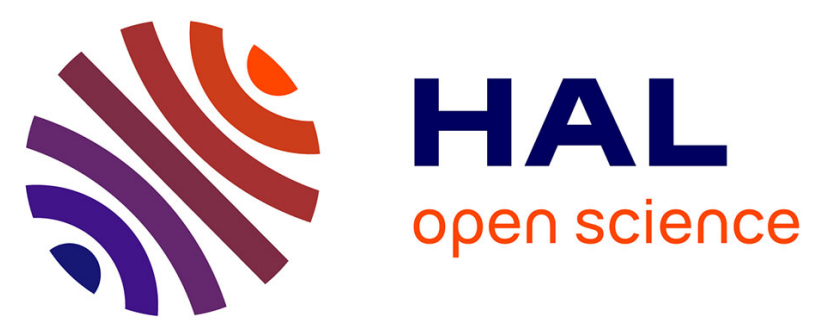

\title{
Networked Cooperation-Based Distributed Model Predictive Control Using Laguerre Functions for Large-Scale Systems
}

Kamel Menighed, Ahmed Chemori, Boumedyen Boussaid, Joseph Juien Yamé

\section{- To cite this version:}

Kamel Menighed, Ahmed Chemori, Boumedyen Boussaid, Joseph Juien Yamé. Networked Cooperation-Based Distributed Model Predictive Control Using Laguerre Functions for Large-Scale Systems. ICEECA 2019 - 4th International Conference on Electrical Engineering and Control Applications, Dec 2019, Constantine, Algeria. 10.1007/978-981-15-6403-1_9 . hal-02420187

\section{HAL Id: hal-02420187 \\ https://hal.science/hal-02420187}

Submitted on 8 Jan 2020

HAL is a multi-disciplinary open access archive for the deposit and dissemination of scientific research documents, whether they are published or not. The documents may come from teaching and research institutions in France or abroad, or from public or private research centers.
L'archive ouverte pluridisciplinaire HAL, est destinée au dépôt et à la diffusion de documents scientifiques de niveau recherche, publiés ou non, émanant des établissements d'enseignement et de recherche français ou étrangers, des laboratoires publics ou privés. 


\section{Networked Cooperation-Based Distributed Model Predictive Control using Laguerre Functions for Large-Scale Systems}

\author{
Kamel Menighed \\ Department of Petrochemicals and process engineering \\ University of 20 août 1955 \\ Skikda, Algeria \\ k.menighed@univ-skikda.dz
}

\author{
Joseph-Julien Yamé \\ CRAN Laboratory \\ University of Lorraine \\ Nancy, France \\ joseph.yame@univ-lorraine.fr
}

\author{
Ahmed Chemori \\ Boumedyen Boussaid \\ University of Montpellier 2 National School of Engineers of Gabès \\ LIRMM-CNRS \\ Montpellier, France \\ Ahmed.Chemori@lirmm.fr
}

\begin{abstract}
This paper proposes a novel cooperative distributed control system architecture based on unsupervised and independent Model Predictive Control (MPC) using discretetime Laguerre functions to improve the performance of the whole system. In this distributed framework, local MPCs algorithms might exchange and require information from other sub-controllers via the communication network to achieve their task in a cooperative way. In order to reduce the computational burden in the local rolling optimization with a sufficiently large prediction horizon, the orthonormal Laguerre functions are used to approximate the predicted control trajectory. Simulation results show that the proposed architecture could guarantee satisfactory global performance even under strong interactions among the subsystems.
\end{abstract}

Keywords-large-scale interconnected systems, distributed model predictive control, Laguerre functions.

\section{INTRODUCTION}

During the past two decades a lot of interest has been given to the control of a class of large-scale systems which are composed by many physically or geographically divided subsystems. However, these subsystems are interconnected and characterized by significant interactions. Each subsystem interacts with the other subsystems through their states and their inputs. At the same time, due to the high performance requirements, modern control systems are becoming more and more complex. For these processes, different control solutions have be developed to achieve some global performance of the whole system.

In centralized MPC architecture, a control agent is able to acquire the information of the global system and all the input sequences are optimized with respect to one given performance index in a single optimization problem. However, when the number of the state variables and inputs of the system becomes larger, the computation burden of the centralized optimization problem may increase significantly. Therefore, it is often impractical to apply the centralized MPC to largescale systems due to their size and their higher risk of failure of the MPC controller due to its centralized nature. However, the centralized control scheme in these industrial process has gradually given way to decentralized and distributed control strategies for their remarkable decrease in system dimensionality using the local MPC control [1], [7].

The main idea in both decentralized and distributed control in large interconnected systems is to approximate the overall system with several separated subsystem models, where each subsystem is controlled by a local controller. Indeed, each local controller uses the so-called local performance index. On the one hand, these control architectures have the advantages of being flexible to system structure, error-tolerance, less computational efforts and no global information requirements [11], [18], [19]. The only difference between them is that distributed control schemes give due consideration to the external state and/or control signal information communicated between subcontrollers from other subsystems while decentralized ones follow a completely decoupled approach [2], [4], [17].

On the other hand, the development of DCS (Distributed Control Systems), field-bus, communication network technologies in process industries allows the control methodologies to utilize their potentials for improving control performance. In this case, the improvement of the global control performance of the entire system using the network information exchange for large-scale systems is a valuable open problem.

In order to fulfill the global objective for the global system, cooperation between the controllers through a digital communication network might be necessary. Thanks to the digital network, the required cooperation can be achieved by means of a proper information exchange between the subcontrollers. From the control point of view, it is well known 
that MPC allows to deal with linear, nonlinear, multivariable and constrained systems [3]. Moreover, MPC technique benefits greatly from both advances in communication technology and advances in computational resources.

Recalling that the MPC strategy is based on an on-line optimization problem and uses a process model to predict the effect of the control sequence on the behavior of the plant. Typically, MPC is implemented in a centralized way. The complete system is modeled and all the control inputs are computed in one optimization problem. However, for large interconnected systems, it may be necessary to have a distributed control scheme as mentioned above, where local control inputs are computed using local measurements and small order models of the local dynamics. But with information exchange between the controllers, the objective is to achieve some degree of cooperation between sub-controllers that are solving MPC problem with locally relevant variables, costs and constraints.

Previous works on distributed MPC are reported in [6], [13]-[15]. A preliminary analysis of the control performance of distributed MPC has been addressed in [13]. Menighed et al [6] and Razavinasab et al [10] proposed a distributed state estimation strategy, developed for supporting distributed state feedback MPC for large-scale interconnected systems. In [15], two approaches for a coordination between sub-controllers are proposed leading to the so-called communication and cooperation based MPC. In the cooperation-based MPC, each sub-controller knows the global objective in order to improve optimality and stability and makes the decentralized strategy very close to the centralized one. When only the local objectives are known, a hierarchical decentralized control architecture uses a supervisor to compute the global optimum and to coordinate the sub-controllers, in communication-based MPC [8].

In this paper, we deal with unconstrained distributed model predictive control of large-scale interconnected systems to achieve global performance based on the use of a cooperative strategy between the sub-controllers. Thanks to the flexibility and the on-line optimization process inherent to MPC algorithms, we propose a distributed framework based on MPC with Laguerre functions. In order to reduce the computational burden in the local rolling optimization with a sufficiently large prediction horizon, the orthonormal Laguerre functions are utilized to approximate the predicted control trajectory. It is worth to emphasize that the MPC/Laguerre approach is becoming an important topic in modern control theory and practice [16].

\section{Complex Process Interaction Modeling}

We consider the overall system represented as a discrete, linear time-invariant (LTI) model of the form

$$
S \triangleq\left\{\begin{aligned}
x(k+1) & =A x(k)+B u(k) \\
y(k) & =C x(k)
\end{aligned}\right.
$$

where $x(k) \in \Re^{n_{x}}, u(k) \in \Re^{n_{u}}$ and $y(k) \in \Re^{n_{y}}$ are the state, the control input and the output, respectively with their corresponding dimensions, $n_{x}, n_{u}, n_{y}$.

In the decentralized modeling framework, it is assumed that subsystem-subsystem interactions have a negligible effect on system variables, i.e., we suppose that the previous overall model $S$ is composed of $N$ subsystems $S_{i i}$, each subsystem is represented by the following state-space equations

$$
S_{i i} \triangleq\left\{\begin{aligned}
x_{i}(k+1) & =A_{i i} x_{i}(k)+B_{i i} u_{i}(k) \\
y_{i}(k) & =C_{i i} x_{i}(k) \quad i=1,2, \ldots, N
\end{aligned}\right.
$$

where $x_{i}(k) \in \Re^{n_{x_{i}}}, u_{i}(k) \in \Re^{n_{u_{i}}}$ and $y_{i}(k) \in \Re^{n_{y_{i}}}$ are the local state, control input and output, respectively and $n_{x}=\sum_{i} n_{x_{i}}, n_{u}=\sum_{i} n_{u_{i}}$ and $n_{y}=\sum_{i} n_{y_{i}}$. Frequently, the components of the interconnected system are tightly coupled due the material/energy and/or information flow between them. In such cases, the decentralized assumption leads to a loss in achievable control performance. It is natural to view the previous overall model $S$ composed of $N$ subsystems $S_{i}$ interacting with each other through linear interconnections. Each subsystem is represented by the following state-space equations

$$
S_{i} \triangleq\left\{\begin{aligned}
x_{i}(k+1) & =A_{i i} x_{i}(k)+B_{i i} u_{i}(k)+w_{i}(k) \\
y_{i}(k) & =C_{i i} x_{i}(k)+v_{i}(k) \quad i=1,2, \ldots, N
\end{aligned}\right.
$$

where the state and output interaction vectors $w_{i}$ and $v_{i}$ are given by

$$
\begin{aligned}
w_{i}(k) \triangleq \sum_{j=1 ; j \neq i}^{N} A_{i j} x_{j}(k)+\sum_{j=1 ; j \neq i}^{N} B_{i j} u_{j}(k) \\
v_{i}(k) \triangleq \sum_{j=1 ; j \neq i}^{N} C_{i j} x_{j}(k)
\end{aligned}
$$

These vectors represent the interaction of subsystem $j \neq i$ on subsystem $i$. The proposed distributed control architecture fosters implementation of cooperation-based strategy for several interacting processes (3)-(4) in order to emulate the benefits achievable with centralized control.

\section{PRoblem Statement}

By means of (3) and (4), the future state and output prediction over a horizon $p$ are given by

$$
\begin{aligned}
\hat{x}_{i}(k+l \mid k)= & A_{i i}^{l} \hat{x}_{i}(k \mid k)+\sum_{s=1}^{l} A_{i i}^{s-1} B_{i i} u_{i}(k+l-s \mid k) \\
& +\sum_{s=1}^{l} A_{i i}^{s-1} \hat{w}_{i}(k+l-s \mid k-1) \\
\hat{y}_{i}(k+l \mid k)= & C_{i i} \hat{x}_{i}(k+l \mid k)+\hat{v}_{i}(k+l \mid k-1)
\end{aligned}
$$

Given the overall system $S$ composed by $N$ interactive subsystems $S_{i}, i=1,2, \ldots, N$, the unconstrained Distributed Model Predictive Control (DMPC) problem with prediction horizon $p>0$ and control horizon $m>0$ two integer values, with $m \leq p$ consists of finding, at time $k$, a set of independent sub-controllers $\mathcal{C}_{i}$ such that each $\mathcal{C}_{i}$ minimizes the local cost function $J_{i}$. 
$J_{i}=\sum_{l=1}^{p}\left\|y_{i}^{d}(k+l \mid k)-\hat{y}_{i}(k+l \mid k)\right\|_{Q_{i}}^{2}+\sum_{l=1}^{m}\left\|\Delta u_{i}(k+l-1 \mid k)\right\|_{R_{i}}^{2}$

$$
\text { for } i=1, \ldots, N
$$

subject, for $l=1, \ldots, p$, to $\left\{\begin{array}{l}\text { model constraints given by }(5) \\ \text { initial condition }: \hat{x}_{i}(k \mid k)=\hat{x}_{i}(k)\end{array}\right.$

where

$\|\alpha\|_{\Lambda}^{2 \triangleq} \triangleq \alpha^{T} \Lambda \alpha$ is the norm of vector $\alpha$ induced by matrix $\Lambda$. $y_{i}^{d}(k+l \mid k):$ the desired output.

$\Delta u_{i}(k+l-1 \mid k)$ : future control increment at time $k$, with $u_{i}(k)=u_{i}(k-1)+\Delta u_{i}(k \mid k)$.

$Q_{i}$ : a symmetric and positive semi-definite matrix of appropriate dimension $\left(Q_{i} \geq 0\right)$.

$R_{i}$ : a symmetric and positive definite matrix of appropriate dimension $\left(R_{i}>0\right)$.

It should be noted that $\Delta u_{j}(k+l-1 \mid k)$ is excluded in the performance index, since it is independent of the future control sequence of $S_{j}$.

\section{COOPERATION BASED DISTRIBUTED MPC PROBLEM}

In order to find an explicit solution to the first version of DMPC problem, each sub-controller $\mathcal{C}_{i}$ is decomposed into three connected function blocks: an optimizer, a state predictor and an interaction predictor.

\section{Assumptions 1}

The following assumptions are considered:

- the prediction and control horizons are the same for all sub-controllers, i.e., $m_{i}=m_{j}=, p_{i}=p_{j}=p$, $\forall i, j=1,2, \ldots, N, j \neq i$

- all the sub-controllers are synchronous;

- all the sub-controllers communicate only once within a sampling interval;

- all the communication channel introduces a delay of one sampling period.

To simplify the mathematical expressions, the following notations are adopted

- $0_{a \times b}$ is the $a \times b$ null matrix ;

- $I_{a}\left(0_{a}\right)$ is the $a \times a$ identity (null) matrix;

- $\operatorname{diag}_{a}\{\mathcal{A}\}$ is a diagonal block matrix made by $a$ blocks equal to $\mathcal{A}$;

1) Interaction prediction: Under assumptions 1 , at step $k$, the predictions of the interaction vectors are given by

$$
\begin{aligned}
& \hat{W}_{i}(k, p \mid k-1)=\tilde{A}_{i} \hat{X}(k, p \mid k-1)+\tilde{B}_{i} \tilde{\Gamma}_{i} U(k-1, m \mid k-1) \\
& \hat{V}_{i}(k, p \mid k-1)=\tilde{C}_{i} \hat{X}(k, p \mid k-1) \\
& \tilde{H}_{i} \triangleq\left[\operatorname{diag}_{p}\left\{H_{i, 1}\right\} \ldots \operatorname{diag}_{p}\left\{H_{i, i-1}\right\} \quad 0 \quad \operatorname{diag}_{p}\left\{H_{i, i+1}\right\}\right. \\
& \left.\ldots \operatorname{diag}_{p}\left\{H_{i, N}\right\}\right]
\end{aligned}
$$

where $\tilde{H}_{i} \in\left\{\tilde{A}_{i}, \tilde{B}_{i}, \tilde{C}_{i}\right\}$
2) State predictor: Under assumptions 1, at step $k$, the local state prediction for the sub-controller $\mathcal{C}_{i}$ is expressed by

$$
\begin{array}{r}
\hat{x}_{i}(k+l \mid k)=A_{i i}^{l} \hat{x}_{i}(k \mid k)+\sum_{s=1} A_{i i}^{s-1} B_{i i} u_{i}(k+l-s \mid k) \\
+\sum_{s=1}^{l} A_{i i}^{s-1} \hat{w}_{i}(k+l-s \mid k-1)
\end{array}
$$

3) Optimal Control Sequence: Under assumptions 1, at step $k$, based on the exchanged information, the interaction prediction together with the local measurement is used by the optimizer to solve the MPC optimization problem (without constraints). Once computed the optimal control sequence $\left[\Delta u_{i}(k \mid k), \ldots, \Delta u_{i}(k+m-1 \mid k)\right]$, which minimize the local cost function (6), only the first element of the optimal sequence $\Delta u_{i}(k \mid k)$ is selected and the control action $u_{i}(k)=u_{i}(k-1)+\Delta u_{i}(k \mid k)$ is computed and applied as control action to the subsystem $S_{i}$, following the receding horizon strategy. Generally, the parameters of the above optimization problem are $p$ and $m$; these parameters are directly affecting computational load in MPC. One of the MPC formulation is the classic approach presented in [5]. In this approach, for the case of rapid sampling, complicated process dynamics and/or high demands on closed-loop performance, satisfactory approximation of the control signal $\Delta u(k)$ may require a very large number of parameters (large $m$ ), leading to poorly numerically conditioned solutions and heavy computational load when implemented on-line. Instead, a more appropriate technique would be to use Laguerre network in the design of MPC presented in [9], [16].

\section{DESCRIPTION OF THE CONTROL SIGNAL TRAJECTORY}

The Z-transforms of the discrete-time Laguerre networks are written as follows

$$
\Gamma_{n}(z)=\Gamma_{n-1}(z) \frac{z^{-1}-a}{1-a z^{-1}} \quad n=2,3, \ldots, M
$$

with $\Gamma_{1}(z)=\frac{\sqrt{1-a^{2}}}{1-a z^{-1}}$

where $M$ is the number of Laguerre functions in the network and $a$ is the pole of the Laguerre network. The scaling factor $a$ is required to be selected by the user, where $0 \leq a<1$ for the stability of the network. Note that the Laguerre networks are well known for their orthonormality.

With the relation (9), the Laguerre network is illustrated in Fig.1

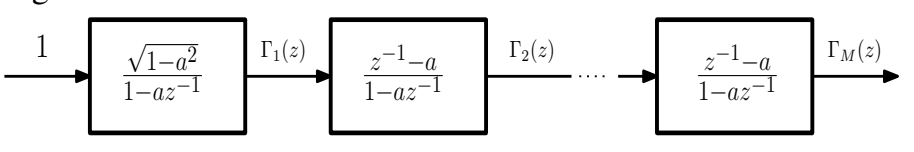

Fig. 1. Illustration of a discrete Laguerre network

The discrete-time Laguerre functions are obtained through the inverse Z-transform of the Laguerre network. The set of Laguerre functions can be expressed as $L(k)=\left[\begin{array}{llll}l_{1}(k) & l_{2}(k) & \ldots & l_{M}(k)\end{array}\right]^{T}$, where $l_{i}(k)$ denotes the inverse Z-transform of $\Gamma_{i}(z, a)$. Taking advantage of the 
network realization (9), the set of discrete-time Laguerre functions satisfies the following difference equation

$$
L(k+1)=\mathcal{A}_{l} L(k)
$$

where $\mathcal{A}_{l} \in \Re^{M \times M}$ and is a function of the parameters $a$ and $\beta=\left(1-a^{2}\right)$, and the initial condition is given by

$$
L(0)^{T}=\sqrt{\beta}\left[\begin{array}{lll}
1-a & a^{2}-a^{3} \ldots(-1)^{M-1} a^{M-1}
\end{array}\right]
$$

The orthonormality can be expressed by

$$
\begin{aligned}
& \sum_{k=0}^{\infty} l_{i}(k) l_{j}(k)=0 \quad \text { for } i \neq j \\
& \sum_{k=0}^{\infty} l_{i}(k) l_{j}(k)=1 \quad \text { for } i=j
\end{aligned}
$$

The orthonormal property of the Laguerre functions will be used in the design of MPC. The key idea in MPC based on Laguerre functions lies in the approximating member of control sequence by a set of Laguerre functions as

$$
\left\{\begin{aligned}
\Delta u(k+l \mid k) & =\sum_{n=1}^{M} l_{n}(l) c_{n}(k)=L(l)^{T} \eta(k) \\
\text { for } l=0,1, \ldots, m-1 & \\
\text { where } & \Delta u(k \mid k)=u(k \mid k)-u(k-1 \mid k)
\end{aligned}\right.
$$

with $k$ being the initial time of the moving horizon window and $l$ being the future sampling. The parameter vector $\eta$ comprises $M$ Laguerre coefficients: $\eta=\left[\begin{array}{llll}c_{1} & c_{2} & \ldots & c_{M}\end{array}\right]^{T}$ and $L(l)^{T}$ is the transposed Laguerre functions vector as defined in the difference equation (10).

By using this approximation, the optimization problem (6) can be expressed in terms of coefficient vector $\eta$, instead of $\Delta u(k)$ as in the classic approach. Thus, the coefficient vector $\eta$ will be optimized and computed in the design. With this design framework, the control horizon $m$ from the classical MPC approach has vanished. Instead, the number of terms $M(M<m)$ is used to describe the complexity of the trajectory in conjunction with the free parameter $a$. Furthermore, a long control horizon $m$ can be achieved without using a large number of parameters, leading to low computational burden and memory storage. In this paper, the MPC based on Laguerre functions is used in the proposed DMPC scheme.

\section{USE OF LAGUERRE FUNCTIONS IN DMPC DESIGN}

\section{A. Modified-State predictor}

1) For SISO subsystem $S_{i}, i=1,2, \ldots, N$ : Under assumptions 1 , at step $k$, the future state prediction over the horizon $p$ are given by

$$
\begin{array}{r}
\hat{x}_{i}(k+l \mid k)=A_{i i}^{l} \hat{x}_{i}(k \mid k)+\sum_{s=1}^{l} A_{i i}^{s-1} B_{i i} u_{i}(k+l-s \mid k) \\
+\sum_{s=1}^{l} A_{i i}^{s-1} \hat{w}_{i}(k+l-s \mid k-1)
\end{array}
$$

let us denote $u_{i}(k)=u_{i}(k-1)+\Delta u_{i}(k)$ and $u_{i}(k+l-s)=$ $u_{i}(k-1)+\sum_{r=0}^{l-s} \Delta u_{i}(k+r)$ for $l=1,2, \ldots, p$. Then, the prediction of the future state variables at time $l$ becomes

$$
\begin{aligned}
& \hat{x}_{i}(k+l \mid k)=A_{i i}^{l} \hat{x}_{i}(k \mid k)+\sum_{s=1}^{l} A_{i i}^{s-1} B_{i i} u_{i}(k-1) \\
& +\sum_{s=1}^{l}\left[\sum_{h=s}^{l} A_{i i}^{l-h} B_{i i}\right] L_{i}(s-1)^{T} \eta_{i}+\sum_{s=1}^{l} A_{i i}^{s-1} \hat{w}_{i}(k+l-s \mid k-1) \\
& \text { for } \quad l=1,2, \ldots, p
\end{aligned}
$$

where the function $\Delta u_{i}(k+s-1)$ is replaced by $L_{i}(s-1)^{T} \eta_{i}$ for $s=1,2, \ldots, l$.

The prediction of the plant output will be

$$
\begin{gathered}
\hat{y}_{i}(k+l \mid k)=C_{i i} A_{i i}^{l} \hat{x}_{i}(k \mid k)+C_{i i} \sum_{s=1}^{l} A_{i i}^{s-1} B_{i i} u_{i}(k-1) \\
+C_{i i} \sum_{s=1}^{l}\left[\sum_{h=s}^{l} A_{i i}^{l-h} B_{i i}\right] L_{i}(s-1)^{T} \eta_{i} \\
+C_{i i} \sum_{s=1}^{l} A_{i i}^{s-1} \hat{w}_{i}(k+l-s \mid k-1)+\hat{v}_{i}(k+l \mid k-1)
\end{gathered}
$$

With this formulation, both predictions of state and output variables are expressed in terms of the coefficient vector $\eta_{i}$ of the Laguerre network, instead of $u_{i}$ as in the classical approach. Thus, the coefficient vector $\eta_{i}$ will be optimized and computed in the control design. To compute the prediction, the convolution sum

$$
S_{c i}(l)=\sum_{s=1}^{l}\left[\sum_{h=s}^{l} A_{i i}^{l-h} B_{i i}\right] L_{i}(s-1)^{T}
$$

needs to be computed. To this end, note that

$$
\begin{aligned}
S_{c i}(1)= & B_{i i} L_{i}(0)^{T} \\
S_{c i}(2)= & {\left[A_{i i} B_{i i}+B_{i i}\right] L_{i}(0)^{T}+B_{i i} L_{i}(1)^{T} } \\
= & {\left[A_{i i}+I\right] B_{i i} L_{i}(0)^{T}+B_{i i} L_{i}(0)^{T} \mathcal{A}_{l i}^{T} } \\
= & S_{c i}(1)+A_{i i} S_{c i}(1)+S_{c i}(1) \mathcal{A}_{l i}^{T} \\
S_{c i}(3)= & S_{c i}(2)+A_{i i}^{2} S_{c i}(1)+A_{i i} S_{c i}(1) \mathcal{A}_{l i}^{T}+S_{c i}(1)\left(\mathcal{A}_{l i}^{2}\right)^{T} \\
S_{c i}(4)= & S_{c i}(3)+A_{i i}^{3} S_{c i}(1)+A_{i i}^{2} S_{c i}(1) \mathcal{A}_{l i}^{T}+A_{i i} S_{c i}(1)\left(\mathcal{A}_{l i}^{2}\right)^{T} \\
& +S_{c i}(1)\left(\mathcal{A}_{l i}^{3}\right)^{T}
\end{aligned}
$$

Continuing the recursion in (17) reveals that for $l=2,3, \ldots, p$

$$
\left\{\begin{array}{l}
S_{c i}(l)=S_{c i}(l-1)+\sum_{h=1}^{l} A_{i i}^{h-1} S_{c i}(1)\left(\mathcal{A}_{l i}^{(l-h)}\right)^{T} \\
\text { with } S_{c i}(1)=B_{i i} L_{i}(0)^{T}
\end{array}\right.
$$

The difference equation $L_{i}(k+1)=\mathcal{A}_{l i} L_{i}(k)$ is used for generating the set of Laguerre functions, where $\mathcal{A}_{l i} \in \Re^{M_{i} \times M_{i}}$ and is a function of the parameters $a_{i}$ and $\beta_{i}=\left(1-a_{i}^{2}\right)$.

2) For MIMO subsystem $S_{i}, i=1,2, \ldots, N$ : To extend the description to MIMO subsystem, with full flexibility in the choice of $a_{i}$ and $M_{i}$ parameters, let $\Delta u_{i}(k)$ be

$$
\Delta u_{i}(k)=\left[\begin{array}{ll}
\Delta u_{i}^{1}(k) & \Delta u_{i}^{2}(k) \cdots \Delta u_{i}^{n_{u_{i}}}(k)
\end{array}\right]^{T}
$$


and the input matrix be partitioned to

$$
B_{i i}=\left[\begin{array}{llll}
B_{i i}^{1} & B_{i i}^{2} & \cdots & B_{i i}^{n_{u_{i}}}
\end{array}\right]
$$

where $n_{u_{i}}$ is the number of inputs and $B_{i i}^{g}$ for $g=$ $1,2, \ldots, n_{u_{i}}$ is the $g^{t h}$ column of the $B_{i i}$ matrix. We express the $g^{t h}$ control signal $\Delta u_{i}^{g}(k)$ by choosing a scaling factor $a_{i}^{g}$ and order $M_{i}^{g}$, where $a_{i}^{g}$ and $M_{i}^{g}$ are selected for this particular input, such that

$$
\Delta u_{i}^{g}(k)=L_{i}^{g}(k)^{T} \eta_{i}^{g} \quad \text { for } g=1,2, \ldots, n_{u_{i}}
$$

where $\eta_{i}^{g}$ and $L_{i}^{g}(k)$ are the Laguerre network description of the $g^{t h}$ control, namely

$$
L_{i}^{g}(k)^{T}=\left[\begin{array}{llll}
l_{i(1)}^{g}(k) & l_{i(2)}^{g}(k) & \ldots & l_{i\left(M_{i}^{g}\right)}^{g}(k)
\end{array}\right]
$$

Based on the partition of the input matrix and given the state variable information at $\hat{x}_{i}(k)$, the prediction of the future state at time $l$ can be written as

$\hat{x}_{i}(k+l \mid k)=A_{i i}^{l} \hat{x}_{i}(k \mid k)+\Psi_{i}(l) u_{i}(k-1)+\Phi_{i}(l)^{T} \eta_{i}+\Omega_{i}(l)$

with

$$
\begin{aligned}
& \Psi_{i}(l)=\sum_{s=1}^{l} A_{i i}^{s-1} B_{i i}, \Phi_{i}(l)^{T}=\sum_{s=1}^{l}\left[\sum_{h=s}^{l} A_{i i}^{l-h} B_{i i}\right] L_{i}(s-1)^{T} \\
& \Omega_{i}(l)=\sum_{s=1}^{l} A_{i i}^{s-1} \hat{w}_{i}(k+l-s \mid k-1)
\end{aligned}
$$

where the parameter vector $\eta_{i}$ and the data matrix $\Phi_{i}(l)^{T}$ consist of the following individual coefficient vectors

$$
\eta_{i}^{T}=\left[\begin{array}{ll}
\eta_{i}^{1} & \eta_{i}^{2} \ldots \eta_{i}^{n_{u_{i}}}
\end{array}\right]^{T}
$$

$\Phi_{i}(l)^{T}=\sum_{s=1}^{l}\left[\sum_{h=s}^{l} A_{i i}^{l-h}\left[B_{i i}^{1} L_{i}^{1}(s)^{T} B_{i i}^{2} L_{i}^{2}(s)^{T} \ldots B_{i i}^{n_{u_{i}}} L_{i}^{n_{u_{i}}}(s)^{T}\right]\right]$

for $i=1,2, \ldots, N$ and $g=1,2, \ldots, n_{u_{i}}$.

Note that the $k^{\text {th }}$ block matrix

$$
\Phi_{i}^{k}(l)^{T}=\sum_{s=1}^{l} \sum_{h=s}^{l} A_{i i}^{l-h} B_{i i}^{k} L_{i}^{k}(s-1)^{T}
$$

has an identical structure as the single-input case defined by $S_{c i}(l)$, thus it can be computed recursively using (18). From here on, the convolution sum in each multi-input subsystem $S_{i}$ is decomposed into computing the subsubsystems, and the computed results are put together block by block to form the multi-input structure. It is worth stressing that in the formulation of the multivariable problem, the scaling factors $a_{i}^{g}$ and the number of terms $M_{i}^{g}$ can be chosen independently for each input signal of subsystem $S_{i}$.

\section{B. Modified-Optimizer}

Under assumptions 1, since the discrete Laguerre functions are orthonormal for a sufficiently large prediction horizon $p$, the cost function (6) is equivalent to the modified local cost function defined as

$J_{i}=\sum_{l=1}^{p}\left\|y_{i}^{d}(k+l \mid k)-\hat{y}_{i}(k+l \mid k)\right\|_{Q_{i}}^{2}+\eta_{i}^{T} R_{L_{i}} \eta_{i}$

where $R_{L_{i}} \in \Re^{M_{i} \times M_{i}}$ is a diagonal matrix with the weighting matrix $R_{i}$ on its diagonal. Therefore, the objective is to find the coefficient vector $\eta_{i}$ that minimizes $J_{i}$. Without any constraints, by substituting (15) into (20), the optimal solution of the parameter vector $\hat{\eta}_{i}$ is

$$
\begin{gathered}
\hat{\eta}_{i}=\eta_{i}^{o p t}=\left(\sum_{l=1}^{p} \bar{\Phi}_{i}(l) Q_{i} \bar{\Phi}_{i}(l)^{T}+R_{L_{i}}\right)^{-1} \\
\times\left(\sum _ { l = 1 } ^ { p } \overline { \Phi } _ { i } ( l ) Q _ { i } \left(y_{i}^{d}(k+l \mid k)-\bar{\Theta}_{i}(l) \hat{x}_{i}(k \mid k)-\bar{\Psi}_{i}(l) u_{i}(k-1)\right.\right. \\
\left.\left.-\bar{\Omega}_{i}(l)-\hat{v}_{i}(k+l \mid k-1)\right)\right)
\end{gathered}
$$

with

$$
\begin{array}{rlrl}
\bar{\Theta}_{i}(l) & =C_{i i} A_{i i}^{l}, & \bar{\Psi}_{i}(l)=C_{i i} \Psi_{i}(l) \\
\bar{\Phi}_{i}(l)^{T} & =C_{i i} \Phi_{i}(l)^{T}, & & \bar{\Omega}_{i}(l)=C_{i i} \Omega_{i}(l)
\end{array}
$$

Upon obtaining the optimal parameter vector $\hat{\eta}_{i}$, the control increment at time $k$ is as follows

$$
\Delta u_{i}(k)=\left[\begin{array}{cccc}
L_{i}^{1}(0)^{T} & 0_{2}^{T} & \ldots & 0_{n_{u_{i}}}^{T} \\
0_{1}^{T} & L_{i}^{2}(0)^{T} & \ldots & 0_{n_{u_{i}}}^{T} \\
\vdots & \vdots & \ddots & \vdots \\
0_{1}^{T} & 0_{2}^{T} & \ldots & L_{i}^{n_{u_{i}}}(0)^{T}
\end{array}\right] \hat{\eta}_{i}
$$

where $0_{r}^{T}$, for $r=1,2, \ldots, n_{u_{i}}$, represents a zero block row vector with identical dimension of $L_{i}^{r}(0)^{T}$.

Consequently, the control signal $u_{i}(k)$ can be calculated as

$$
u_{i}(k)=u_{i}(k-1)+\Delta u_{i}(k)
$$

1) Modified Interaction prediction: Under assumptions 1, at step $k$, the predictions of the new interaction vectors are given by

$$
\begin{aligned}
& \hat{W}_{i}(k, \mid k-1)=\tilde{A}_{i} \hat{X}(k \mid k-1)+\tilde{B}_{i} \tilde{U}(k-1 \mid k-1) \\
& \hat{V}_{i}(k, \mid k-1)=\tilde{C}_{i} \hat{X}(k \mid k-1) \\
& \tilde{F}_{i} \triangleq\left[\begin{array}{lllllll}
F_{i, 1} & \ldots & F_{i, i-1} & 0 & F_{i, i+1} & \ldots & F_{i, N}
\end{array}\right]
\end{aligned}
$$

where $\tilde{F}_{i} \in\left\{\tilde{A}_{i}, \tilde{B}_{i}, \tilde{C}_{i}\right\}$

\section{NC-MPC ALGORITHM}

For the $i^{\text {th }}$ sub-controller $\mathcal{C}_{i}$, where the desired output $y_{i}^{d}(k+l \mid k)$ is provided by a proper reference generator, the algorithm for the novel distributed MPC is outlined in detail:

1) Set $k=1$

2) Acquire by network the predicted future state trajectories $\hat{X}_{j}(k \mid k-1)$ and control inputs $U_{j}(k-1 \mid k-1)$ from sub-controllers $\mathcal{C}_{j}$. 
3) Build $\hat{X}(k \mid k-1)$ and $U(k-1 \mid k-1)$ by combining the local state trajectory $\hat{x}_{i}(k \mid k-1)$ and control input $u_{i}(k-1 \mid k-1)$ with the acquired information, and compute the corresponding predictions of the interactions according to (24).

4) Acquire the measures $\hat{x}_{i}(k)$ and the desired trajectory $y_{i}^{d}(k+l \mid k)$ over the horizon $p$.

5) Compute the optimal control $u_{i}(k)$ and broadcast it by network to sub-controllers $\mathcal{C}_{j}$, cf. (21), (22) and (23).

6) Apply the control input $u_{i}(k)$ to subsystem $S_{i}$.

7) Compute the future state trajectory of subsystem $S_{i}$ over the horizon $p$ and broadcast it by network to subcontrollers $\mathcal{C}_{j}$, cf. (19).

8) Increment the sample time index $k \leftarrow k+1$ and iterates by going to step 2 .

\section{Simulation AND RESUlts}

In this section, the performance of the proposed NC-DMPC is investigated and compared to the centralized MPC. Consider the following unstable-non minimum phase plant $S$ introduced in [12] discretized with a sampling time $T_{s}=0.2 \mathrm{~s}$. A statespace realization of $S$ has the form (1), with the following matrices

$A=\left[\begin{array}{cc}A_{11} & 0 \\ 0 & A_{22}\end{array}\right], B=\left[\begin{array}{cc}B_{11} & 0 \\ 0 & B_{22}\end{array}\right], C=\left[\begin{array}{ll}C_{11} & C_{12} \\ C_{21} & C_{22}\end{array}\right]$

The examination of the process model $S$ leads to decomposition into two interconnected subsystems $S_{1}$ and $S_{2}$. The corresponding state-space realizations of $S_{1}$ and $S_{2}$ have the form (2), with matrices $\left\{A_{11}, B_{11}, C_{11}\right\}$ and $\left\{A_{22}, B_{22}, C_{22}\right\}$, respectively. The constant parameter $\alpha$ is used to study the effect of the interactions between $S_{1}$ and $S_{2}$.

$A_{11}=\left[\begin{array}{cccc}2.859 & -1.335 & 0.409 & 0 \\ 2 & 0 & 0 & 0 \\ 0 & 1 & 0 & 0 \\ 0 & 0 & 0 & 0.819\end{array}\right], B_{11}=\left[\begin{array}{c}0.063 \\ 0 \\ 0 \\ 0.125\end{array}\right]$

$A_{22}=\left[\begin{array}{ccc}0.819 & 0 & 0 \\ 0 & 1.637 & -0.67 \\ 0 & 1 & 0\end{array}\right], B_{22}=\left[\begin{array}{c}0.125 \\ 0.250 \\ 0\end{array}\right]$

$C_{11}=\left[\begin{array}{ll}-0.0153-0.029-0.007 & 0\end{array}\right], C_{12}=\alpha\left[\begin{array}{lll}0.145 & 0 & 0\end{array}\right]$

$C_{22}=\left[\begin{array}{lll}0 & -0.193 & 0.292\end{array}\right], C_{21}=\alpha\left[\begin{array}{llll}0 & 0 & 0 & 0.145\end{array}\right]$
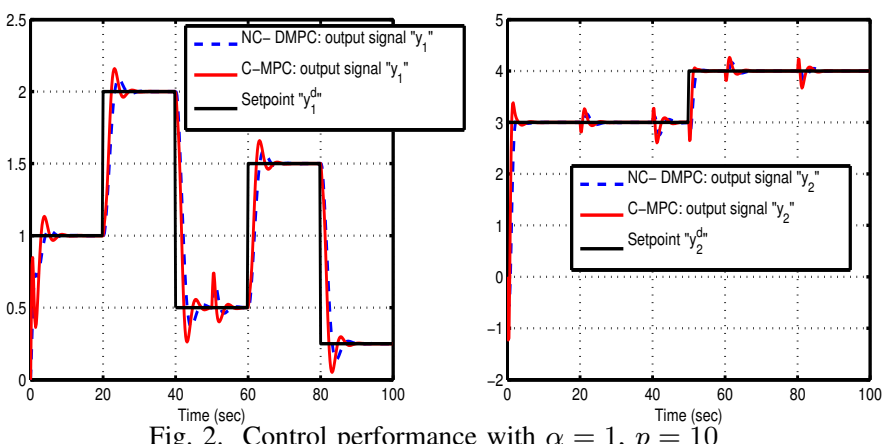

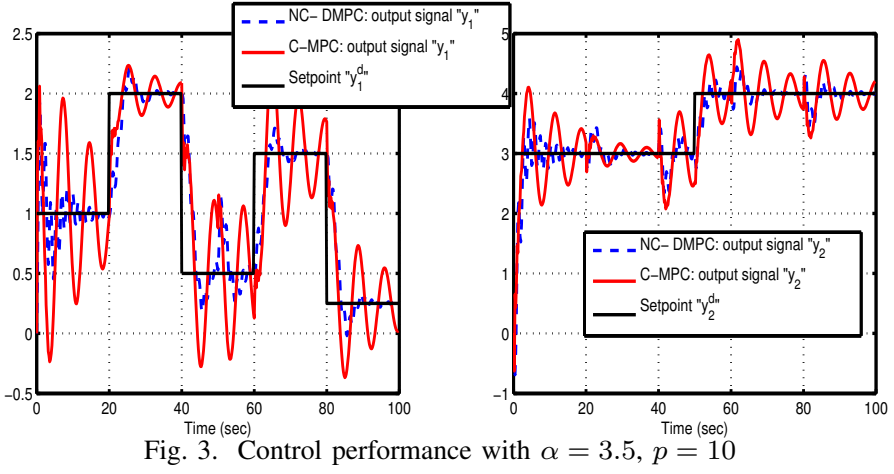

In the proposed test, the stability performances depend on the choice of the tuning parameters $\alpha$ and $p$. The control performance of the resulting closed-loop system is plotted in Figs 2, and 3, where the black lines correspond to the desired outputs, the red solid lines correspond to the system outputs using Centralized MPC (C-MPC) based on Laguerre functions, and the blue solid lines represent the system outputs using the proposed NC-DMPC. In Fig 2, the performance of NDDMPC is comparable to the one of C-MPC. However, for strong interactions with $\alpha=3.5$, Fig 3 shows that the NDDMPC strategy can achieve a better global performance of the closed-loop system than C-MPC.

To sum up, for the given example, the NC-DMPC can achieve a satisfactory global performance even whether the interactions among subsystems are strong or not. Furthermore, the cost of computation is very small as compared with the classical centralized controller.

\section{CONCLUSION}

In the present study, a formulation of a novel distributed model predictive control for a class of large-scale systems is proposed, in which the whole system is divided into many small scale subsystems interacting with each other by both their states and inputs. The NC-DMPC solution, was proposed for improving the global performance of closed-loop system; it is based on Laguerre functions used in MPC formulation. The proposed methodology is demonstrated on an example for a set-point tracking. Compared to the C-MPC scheme, the proposed NC-DMPC allowed to achieve an improved performance of the whole system through using a local index in optimization.

\section{REFERENCES}

[1] P.D. Christofides, R. Scattolini, D. Muñoz de la Peña and J. Liu, "Distributed Model Predictive control: A Tutorial Review and Future Research Directions", Computers and Chemical Engineering, vol. 51, pp. 21-41, 2013.

[2] M. Ikeda and D.D. Šiljak, "Overlapping Decentralized Control with Input State and Output Inclusion", Control Theory and Advanced Technology, vol. 2, pp. 155-172, 1986.

[3] D. Jia and B.H. Krogh, "Distributed Model Predictive Control", in Proceeding of the American Control Conference, Arlington, pp. $2767-$ 2771, 2001.

[4] J. Lunze, "Feedback Control of Large-Scale Systems", Prentice-Hall, London, UK, 1992.

[5] J. M. Maciejowski, "Predictive control with constraints". London, U.K: Prentice-Hall, 2002. 
[6] K. Menighed, C. Aubrun and J.J. Yamé, 'Distributed State Estimation and Model Predictive Control : Application to Fault Tolerant Control", 7th IEEE International Conference on Control \& Automation, pp. 93694, 2009.

[7] R. R. Negenborn and J. M. Maestre, "Distributed Model Predictive Control: An Overview and Roadmap of Future Research Opportunities", IEEE Control System, vol. 34(4), pp. 87-97, 2014.

[8] R.J. Patton C. Kambhampati and F.J. Uppal, "Challenges of Networked Control Systems: Autonomy, Reconfiguration and Plug and Play", in Proceeding 1st Workshop on Network Control Systems and FaultTolerant Control, Ajaccio, Corsica, 2005.

[9] X. Qian, Y. Yin, X. Zhang, X. Sun and H. Shen, "Model Predictive Controller Using Laguerre Functions for Dynamic Positioning System", 35th Chinese Control Conference, pp. 4436-4441, 2016.

[10] Z. Razavinasab, M.M. Farsangi and M. Barkhordari, "State Estimationbased Distributed Model Predictive Control of Large-Scale Networked Systems with Communication Delays", IET Control Theory \& Applications, vol. 11(15), pp. 2497-2505, 2017.

[11] M. Vaccarini, S. Longhi and M.R. Katebi, "Unconstrained Networked Decentralized Model Predictive Control", Journal of Process Control, vol. 19(2), pp. 328-339, 2009.

[12] M. Vaccarini, S. Longhi and M.R. Katebi, "Stability Analysis Tool for Tuning Unconstrained Decentralized Model Predictive Controllers", in Proceedings of the International Conference Control, Glasgow, Scotland, UK, August 2006.

[13] M. Vaccarini, S. Longhi and M.R. Katebi, "State Space Analysis of Unconstrained Decentralized Model Predictive Control Systems", in Proceeding of the American Control Conference, Minneapolis, Minnesota, pp. 159-164, 2006.

[14] A.N. Ventak, I.A. Hiskens, J.B. Rawlings and S.J. Wright, "Distributed Output Feedback MPC for Power System Control", In Proceedings of the 45th IEEE Conference on Decision and Control, San Diego, California, pp. 4038-4045, 2006 a.

[15] A.N. Venkat, J.B. Rawlings, and S.J. Wright, "Stability and Optimality of Distributed Model Predictive Control", in Proceedings of the 44th
IEEE Conference on Decision and Control, and the European Control Conference, pp. 6680-6685, 2005.

[16] L. Wang, "Model Predictive Control System Design and Implementation Using MATLAB", Advances in Industrial Control, Springer, 2009.

[17] A.I. Zečević and D.D. Šiljak, "A New Approach to Control Design with Overlapping Information Structure Constraints", Automatica, vol. 41, pp. 265-272, 2005.

[18] Y. Zheng, S. Li and N. Li, "Distributed Model Predictive Control Over Network Information Exchange for Large-Scale Systems", Control Engineering Practice, vol. 19(7), pp. 757-769, 2011.

[19] Y. Zheng, S. Li and H. Qiu, "Networked Coordination-Based Distributed Model Predictive Control for Large-Scale System", IEEE Transactions On Control Systems Technology, vol. 21(3), pp. 991-998, 2013. 